\title{
Babesia bovis: Actualidad del desarrollo de una vacuna
}

\author{
Laura Cuy-Chaparro', Laura A Ricaurte-Contreras², Anny J Camargo-Mancipe', \\ Darwin A Moreno-Pérez ${ }^{3}$
}

\section{RESUMEN}

Introducción. Babesia bovis es el principal agente causal de la babesiosis bovina, una importante enfermedad veterinaria transmitida por garrapatas a nivel mundial. Las estrategias convencionales para controlar esta parasitosis han presentado múltiples limitaciones por lo que el desarrollo de una vacuna basada en antígenos representa una estrategia apropiada para la prevención y el tratamiento.

Objetivo. Describir los aspectos relevantes del ciclo de vida del parásito $B$. bovis, la epidemiología, diagnóstico y la aplicación de diferentes estrategias usadas para controlar esta parasitosis. Además, se discuten potenciales puntos de intervención para desarrollar una vacuna contra este parásito.

Metodología. Se realizó una búsqueda en las bases de datos usando los términos: "Babesia bovis AND lyfe cycle", "B. bovis vaccine and Vaccine candidates", entre otras. Los estudios con mayor pertinencia publicados hasta la actualidad se revisaron completamente.

Resultados. Los detalles de la biología de parásito $B$. bovis y el proceso molecular usado para ocasionar la enfermedad en el hospedador son poco conocidos, lo que explica que el desarrollado de estrategias para el control de esta parasitosis no hayan sido del todo eficientes. Por lo tanto, se requiere diseñar nuevas medidas, por ejemplo, desarrollar vacunas de nueva generación basadas en un enfoque funcional que permitan mejorar las condiciones de sanidad animal.

Conclusiones. Comprender el complejo ciclo de vida de $B$. bovis permitirá estudiar las interacciones huésped-parásito-garrapata e identificar moléculas implicadas en la adhesión/invasión celular para evaluar su utilidad como componente de una vacuna que controle esta parasitosis.

Palabras clave: Babesia bovis, babesiosis, ciclo de vida, vacuna, antígeno.

1 Facultad de Ciencias de la Salud, Universidad de Boyacá, Tunja, Colombia.

2 Universidad Nacional de Colombia, Bogotá, Colombia.

3 Escuela de Medicina y Ciencias de la Salud, Universidad del Rosario. Facultad de Ciencias Agropecuarias, Universidad de Ciencias Aplicadas y Ambientales (U.D.C.A), Bogotá, Colombia.

Correspondencia: Laura Cuy-Chaparro. Dirección: Carrera 2a Este № 64-169 Tunja, Boyacá, Colombia; Celular: 312-502-3782.

Correo electrónico: lecuy@uniboyaca.edu.co.

Citar este artículo así:

Cuy-Chaparro L, Ricaurte-Contreras LA, Camargo-Mancipe AJ, Moreno-Pérez DA. Babesia bovis: Actualidad del desarrollo de una vacuna. Revista Investig Salud Univ Boyacá. 2019;6(2): 182-199. doi: https://doi.org/10.24267/23897325.349 


\title{
Babesia bovis: An Update on vaccine development
}

\begin{abstract}
Introduction. Babesia bovis is the main causal agent of babesiosis bovine, one important veterinary diseases transmitted by ticks worldwide. Conventional strategies to control this parasitosis have shown several limitations and therefore the development of a vaccine will be an appropriate strategy for prevention and treatment.

Objective. To describe relevant aspects of B. bovis parasite's life cycle, the epidemiology, diagnosis, the application of different strategies used to control this parasitosis. In addition, potential points of intervention to develop a vaccine against this parasite has been discussed.

Methodology. A search was made using keywords as "Babesia bovis AND lyfe cycle", "B. bovis vaccine and Vaccine candidates" and others. The most relevant studies published to date were completely revised.

Results. The details of the B.bovis parasite biology and the molecular process used to cause disease in the host had not been describe in deep; explaining that the development of strategies for the control of this parasitosis have not been entirely efficient. Therefore, it is necessary to design new procedures, for example, to develop new generation vaccines based on a functional approach which improve the animal health conditions.
\end{abstract}

Conclusions. Understand the B. bovise's life cycle complex will allow the host-parasite-tick interactions study and the identification of molecules involved in cell adhesion / invasion to evaluate its usefulness as a vaccine component that controls this parasitosis.

Key words: Babesia bovis, babesiosis, life cycle, vaccine, antigen. 


\title{
Babesia bovis: Atualidade do desenvolvimento de uma vacina
}

\begin{abstract}
RESUMO
Introdução. Babesia bovis é o principal agente causador da babesiose bovina, uma importante doença veterinária transmitida por carrapatos a nível mundial. As estratégias convencionais para o controle das parasitoses têm presentado múltiplas limitações pelo que o desenvolvimento de uma vacina baseada em antígenos representa uma estratégia apropriada para a prevenção e o tratamento.

Objetivo. Descrever os aspectos relevantes do ciclo de vida do parasita B. bovis, a epidemiologia, diagnostico e aplicação de diferentes estratégias usadas para o controle desta parasitose. Além disso, são discutidos possíveis pontos de intervenção para o desenvolvimento de uma vacina contra o parasita.
\end{abstract}

Metodologia. Uma pesquisa foi realizada nas bases de dados usando os termos: "Babesia bovis AND Iyfe cycle", "B. bovis vaccine and Vaccine candidates", entre outras. Os estudos mais relevantes publicados até o momento foram completamente revisados.

Resultados. Os detalhes da biologia do parasita B. bovis e o processo molecular usado para causar doenças no hospedeiro é pouco conhecido, o que explica que o desenvolvimento de estratégias para o controle desta parasitose não foram completamente eficientes. Portanto, é necessário projetar novas medidas, por exemplo, desenvolver vacinas de nova geração com base em uma abordagem funcional que permita melhorar as condições de saúde animal.

Conclusões. Compreender o complexo ciclo de vida de B. bovis permitirá estudar as interações hospede-parasita-carrapatos e identificar moléculas envolvidas na adesão/invasão celular para avaliar sua utilidade como componente de uma vacina que controla essa parasitose.

Palavras-chave: Babesia bovis, babesiose, ciclo de vida, vacina, antígeno. 


\section{INTRODUCCIÓN}

Las enfermedades parasitarias representan una de las preocupaciones más comunes para la salud y productividad de animales en varios países del mundo (1). Dentro de estas se encuentra la babesiosis, una de las enfermedades veterinarias más importantes que afecta al ganado bovino, y ocasionalmente a los humanos, en áreas subtempladas y tropicales del mundo (2-4). Esta enfermedad es transmitida por garrapatas, los cuales son vectores que han comenzado a presentar mayor distribución geográfica en el mundo (5-7). La babesiosis bovina es causada por parásitos Apicomplexas del género Babesia (B. bovis, B. bigemina, $B$. divergens y $B$. major); la $B$. bovis es la especie que se asocia con cuadros graves de la enfermedad y mayores pérdidas económicas por la disminución de la producción de carne y leche, el aumento en la morbimortalidad animal y conflictos para el comercio internacional del ganado (8-11).

En Colombia, se ha reportado una prevalencia de babesiosis bovina de $14,4 \%$ (29/202) siendo el $65,5 \%$ causada por $B$. bovis en la región del Valle del río Magdalena y $14 \%$ en Turbo, Antioquía $(12,13)$ bovine cattle and ticks (the transmitting vector. Debido a lo anterior y teniendo en cuenta la adaptación que ha tenido el vector (Rhipicephalus microplus) en diferentes zonas del Altiplano cundiboyacense (14), se plantea un panorama de riesgo para la transmisión de la infección principalmente por $B$. bovis en esta región de importante producción láctea.

El desarrollo de estrategias para el control de estas parasitosis requiere importantes inversiones económicas, dado el elevado costo de prevención (control insecticida del vector) y tratamiento (quimioprofilaxis) de los animales infectados (15). La aparición de resistencia a medicamentos y el desconocimiento de la distribución del vector, han llevado a adoptar como estrategia alterna la vacunación con parásito atenuado, sin embargo, esta es una medida que, además de no estar disponible para uso en todo el mundo, no ha sido del todo eficaz; lo anterior, obliga a usar un método de control con mejor relación costo/ efectividad contra agentes patógenos, como por ejemplo el desarrollo de una vacuna basada en un enfoque funcional, en el cual se incluyen moléculas del parásito conservadas y con un papel importante en el proceso de invasión (16). Para ello, y en el caso de organismos Apicomplexa, se ha propuesto la búsqueda de candidatos vacunales, dirigida a conocer las regiones conservadas y con señales de restricción funcional de aquellos genes codificantes de proteínas relacionadas con la adhesión e invasión de los parásitos a sus células diana (17).

La presente revisión resume aspectos relevantes sobre el complejo ciclo de vida del parásito $B$. bovis, la epidemiología, el diagnóstico, la aplicación de 
diferentes estrategias usadas para el control de esta parasitosis; igualmente, se recoge aquí la utilidad que podría llegar a tener el desarrollo de una vacuna (como alternativa de mayor costo-beneficio) en relación con el control de la Babesiosis, la cual representa un importante riesgo para la industria ganadera nacional.

\section{METODOLOGÍA}

Se realizó una revisión documental para analizar la información conceptual existente hasta el año 2019, en la que se describe la epidemiología, el ciclo de vida de $B$. bovis, la patogénesis, el diagnóstico y el tratamiento de la babesiosis bovina. Se utilizaron fuentes de información primaria como ScienceDirect y Elsevier. Las bases de datos consultadas fueron: PubMed del Centro Nacional de Información Biotecnológica (NCBI), Medline, LILACS y SciELO, utilizando diferentes combinaciones de palabras clave en inglés con el operador booleano AND, entre ellas "(Babesia bovis) AND lyfe cycle", "babesiosis", "tick fever", "transmission", "(B. bovis vaccine) AND Vaccine candidates". Se obtuvieron 320 publicaciones de las cuales fueron seleccionados 59 artículos científicos y 3 publicaciones correspondientes a decretos y comunicaciones de entidades estatales y privadas, disponibles en texto completo en idioma inglés o español, teniendo en cuenta que informaban acerca del ciclo de vida, epidemiología, diagnóstico, tratamiento y estrategias de control de la enfermedad. No se utilizaron otros criterios de selección debido a la limitada información existente acerca del parásito.

\section{Babesiosis: Agente etiológico y vectores}

El phylum Apicomplexa esta constituido por un grupo importante de parásitos intracelulares obligados, como aquellos pertenecientes a los géneros Theileria, Plasmodium y Babesia. Estos parásitos se caracterizan por poseer un complejo apical compuesto de organelos secretores de moléculas (roptrias, micronemas y orgánulos conoides) que participan activamente en el proceso de invasión al eritrocito ocasionando la Teileriosis, Malaria o Babesiosis en el hospedero $(18,19)$.

En el caso de la Babesiosis, esta enfermedad es causada por protozoos del género Babesia. Las especies de mayor importancia que infectan bovinos son Babesia bovis y Babesia bigemina $(2,8)$ mientras que $B$. divergens, $B$. major $B$. ovata, $B$. occultans y $B$. jakimovi son otras Babesias que tiene menor prevalencia (20). El principal vector de parásitos del género Babesia son las garrapatas del género Rhipicephalus spp., e Ixodes spp., que tienen como hospederos definitivos un amplio número de animales domésticos y salvajes; el humano también es un hospedero incidental (21). Los picos de infección y la prevalencia de la babesiosis varían según la condición estacional, 
lo cual depende directamente del ciclo de vida de la garrapata y, por ende, es importante conocerlo para poder establecer estrategias de control integrado apropiadas.

\section{Epidemiología de la Babesiosis}

De acuerdo con la Organización Mundial de Sanidad Animal (OIE), la babesiosis es una enfermedad de declaración obligatoria, dada su importancia socioeconómica y sanitaria, y por las considerables repercusiones que tiene en el comercio internacional de animales y productos provenientes de ellos (22). Esta directriz fue adoptada por Colombia desde el año 2015, de acuerdo con la resolución 003714 emitida por el Instituto Colombiano Agropecuario (ICA) (23). En el contexto internacional, para el año 2017 se reportó un total de 2.518 focos en el mundo (24); asimismo, a nivel nacional se ha reportado una prevalencia de babesiosis bovina de $14,4 \%$, con un $65,5 \%$ por B. bovis, $20,7 \%$ por B. bigemina y $13,8 \%$ de coinfección en la región del Valle del río Magdalena y $14,4 \%$ en Turbo, Antioquía $(12,13)$ bovine cattle and ticks (the transmitting vector. Además de lo anterior, se ha presentado un aumento en la distribución de la garrapata Rhipicephalus microplus en diferentes zonas del altiplano cundiboyacense (14), lo cual plantea un panorama de riesgo para la transmisión de la infección principalmente por $B$. bovis.

\section{Ciclo de vida}

Al igual que ocurre con otros miembros del phylum Apicomplexa (25), B. bovis tiene un ciclo de vida complejo que requiere de un vector donde ocurre la reproducción sexual (garrapatas de la especie $R$. microplus) y distintos hospederos vertebrados donde tiene lugar la multiplicación asexual del parásito (animales y humanos) (Figura 1) (26). La infección del hospedero se presenta por la picadura de garrapatas durante su proceso de alimentación (Figura 1A). En este, se inoculan esporozoítos que invaden eritrocitos a través de interacciones moleculares patógeno-célula; estas favorecen la permeabilidad celular por la alteración de la arquitectura de la membrana de la célula diana (27-29). A diferencia del ciclo de vida de Plasmodium, $B$. bovis no desarrolla una vacuola parasitófora $y$, por ende, se ubica libremente en el citoplasma donde se reproduce por fisión binaria (etapa de merogonia) hasta dar lugar a la formación de dos a cuatro merozoítos (dependiendo de la especie) con capacidad infectiva $(30,31)$ (Figura 1A). Posteriormente, se presenta una lisis celular en donde se liberan los merozoitos los cuales invaden otros eritrocitos gracias a la presencia de proteínas de superficie y moléculas del complejo apical, tales como Merozoite Surface Antigen1 (MSA1), una glicoproteína de 42 kDa distribuida en la superficie del merozoito (29); Rhoptry-Associated Protein 1 (RAP-1), proteína de 60 kDa presente en la superficie apical y roptrias del merozoito (32), 
y Apical Membrane Antigen-1 (AMA-1), proteína integral de membrana de $69 \mathrm{kDa}$ secretada por micronemas (33). El proceso de invasión se presenta de forma continua lo que da lugar a la producción de merogonias sucesivas y asincrónicas $(20,34)$ y por ende, se pueden encontrar distin- tos estadios parasitarios en el torrente sanguíneo del animal (35). El tiempo en que se presentan los signos clínicos puede variar entre 7 a 35 días desde la inoculación del parásito (21).

Figura 1. Ciclo de vida de Babesia bovis. B. bovis. A. B. bovis se transmite a través de la picadura de garrapatas infectadas cuando estas se alimentan. Durante la picadura, los esporozoítos son liberados con la saliva al torrente sanguíneo e invaden directamente los eritrocitos del hospedador permitiendo que los merozoítos se repliquen por fisión binaria e infecten otras células. B. Algunos merozoítos tienen la capacidad de diferenciarse en gametos, que al ser ingeridos por garrapatas se fusionan y convierten en oocinetes móviles a nivel del tracto digestivo, facilitando la infección de tejidos como las glándulas salivales. Por último, a través de una reproducción asexual se generan numerosos kinetos, los cuales se diferencian en esporozoítos que, al ser inoculados al hospedero, perpetúan la infección.

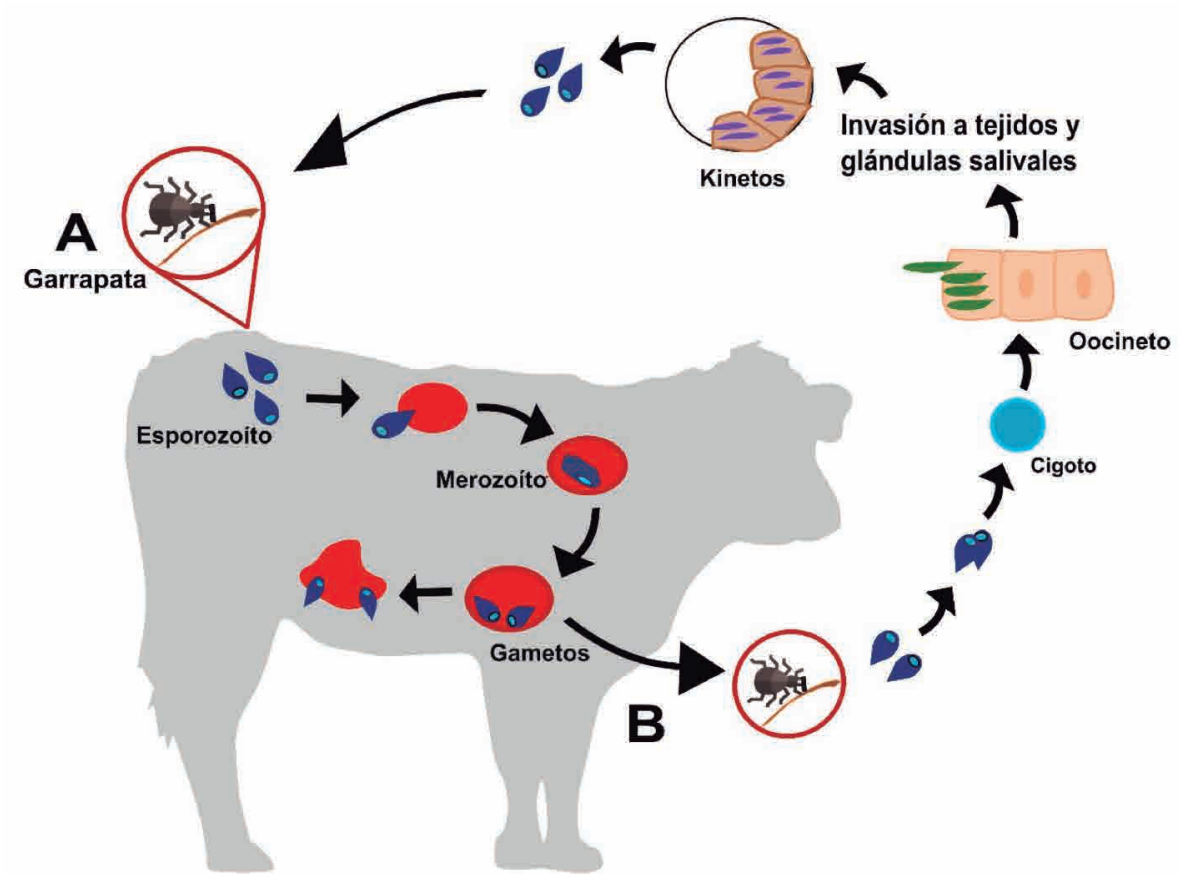


Por otro lado, algunos eritrocitos infectados se desarrollan a formas pre-gametocíticas para dar lugar a los gametocitos (masculinos y femeninos), los cuales son ingeridos por las garrapatas cuando estas se alimentan (Figura 1B). Una vez dentro del vector, los gametos se fusionan en el lumen del tracto digestivo para formar un cigoto alargado de 8 a $10 \mu \mathrm{m}$ de longitud (36). Luego, se presenta una fase de esquizogonia que finaliza con la transformación del parásito en un oocineto móvil, el cual pasa a la hemolinfa e invade tejidos del cuerpo del insecto, incluyendo las glándulas salivales y los ovarios; esta última favorece la transmisión transovárica y trans-estadial (37-40), la cual es una estrategia de perpetuación utilizadas por $B$. bovis (36). Finalmente, se presenta un proceso de multiplicación asexual por esporogonia, generando numerosos kinetos, los cuales invaden las glándulas salivales de los vectores. En esta etapa conocida como infecciosa, se producen esporozoítos, los cuales son inoculados a un hospedero mamífero, de manera que se perpetúe el ciclo de vida (20). Vale la pena destacar, que el estadio transmisor varía según la especie. Por ejemplo, $B$. bovis es inoculada por las larvas de las garrapatas mientras que $B$. bigemina y $B$. divergens las transmiten las ninfas y adultos (41).

\section{Desarrollo clínico y diagnóstico}

La babesiosis presenta una fase aguda caracterizada por la hemólisis continua y exponencial de eritrocitos que conduce al desarrollo de una anemia hemolítica. Esta anemia va acompañada de reticulocitosis y eritrocitosis inducida por estrés, la cual no es suficiente para reponer la cantidad de células destruidas por el parásito. Como consecuencia de lo anterior, se presentan signos clínicos como fiebre, anorexia, letargo, hemoglobinuria, taquicardia e ictericia (8)are all transmitted by Rhiphicephallus (Boophilus. $B$. bovis puede originar un secuestro de parásitos en los capilares cerebrales dando lugar a un cuadro neurológico más grave, acompañado de convulsiones, hiperestesia y parálisis que conducen a la muerte del ganado $(15,42)$.

El diagnóstico de la babesiosis se basa en la historia epidemiológica de la región, la examinación física del animal y la confirmación por pruebas de laboratorio tales como: diagnóstico directo, molecular y serológico (43). El método de diagnóstico tradicional (directo), ampliamente utilizado, ha sido la examinación microscópica del parásito en sangre por medio de un extendido teñido con Giemsa (21). Sin embargo, a pesar de que es un método económico y rápido de hacer, es poco sensible y específico, y además, depende de la experiencia del personal que hace la identificación. Por otro lado, para la confirmación del diagnóstico molecular, se ha utilizado la técnica Reacción en Cadena de la Polimerasa (PCR), que debido a su alta especificidad y sensibilidad permite la identificación de varias especies de Babesia y la detección 
de niveles de parasitemia por debajo de 0,01\% (1 parásito en 100 glóbulos rojos) $(21,41)$. Pese a lo anterior, no es una prueba apropiada para aplicar en campo, dado que se debe tener una infraestructura básica que a menudo suele ser costosa.

Las pruebas serológicas consisten en identificar la presencia del parásito con el uso de anticuerpos generados contra este durante una infección natural; el más utilizado ha sido la inmunofluorescencia indirecta (IFI, Immunofluorescence Assay), el cual muestra poca especificidad por las reacciones cruzadas frente a distintas especies $y$, además, no permite distinguir un tipo de infección reciente o previa debido a su poca sensibilidad en bajas parasitemias (21). Por otro lado, se encuentra la fijación de complemento ( $C F$, Complement fixation), que es un método usado para detectar anticuerpos contra $B$. bovis y $B$. bigemina y así calificar animales de importación. A diferencia de los anteriores métodos, el ensayo de inmunoabsorción ligado a una enzima (ELISA, Enzyme-Linked ImmunoSorbent Assay) ha sido el más utilizado debido a la especificidad y capacidad para procesar un número considerable de muestras diariamente, aunque tampoco es aplicado directamente en campo (43).

\section{Tratamiento y estrategias de control}

En áreas de alta endemicidad, el control de la babesiosis depende del diagnóstico temprano y la adecuada y rápida administración de medicamentos. Distintos compuestos, tales como azul de tripán, diaceturato de diminazeno (3-5 mg/ $\mathrm{kg})$, imidocarb $(1-3 \mathrm{mg} / \mathrm{kg})$ y amicarbalide han sido utilizados como parte de la terapéutica; sin embargo, debido a varias complicaciones como el cambio de coloración de la carne del animal, los problemas de seguridad en la fabricación, o dado que no han sido aprobados por organismo regulados de U.S.A: administración de drogas y medicamentos, estos se han retirado del mercado (41).

La quimioterapia y quimioprofilaxis son estrategias ampliamente utilizadas en todo el mundo y requieren de métodos estratégicos precisos. Lo ideal es que los compuestos químicos actúen sobre moléculas que participan en vías metabólicas exclusivas del parásito. Sin embargo, lo anterior no ha sido posible y, por ende, la mayoría de los compuestos tienen un efecto tóxico, tanto en el hospedero como en el agente patógeno, siendo más susceptible este último. El Imidocarb, un derivado de carbamilo y medicamento de elección para tratar babesiosis causada por $B$. bovis, $B$. bigemina, $B$. divergens y $B$. caballi en una dosis recomendada de $1-3 \mathrm{mg} / \mathrm{kg}$ ha reportado alta concentración del medicamento en tejidos luego del tratamiento, e incluso en la leche, lo que pone de manifiesto la poca utilidad en animales de producción $(43,44)$. Dentro de los compuestos en proceso de estudio que solo han sido evaluados in vitro se encuentran: 
el triclosán, nerolidol, artesunato, epoxomicina, gossypol y atovacuona (43).

El triclosán inhibe la síntesis de ácidos grasos en las membranas celulares, pero estudios genómicos han indicado que $B$. bovis no posee la vía de síntesis de ácidos grasos lo que sugiere que actúa alterando propiedades físicas de la membrana, razón que hace necesario realizar estudios sobre el modo de acción en la babesiosis bovina (43). El nerolidol interviene en la biosíntesis de isoprenoides en el apicoplasto e inhibe el crecimiento de $B$. bovis por completo a concentraciones de $75 \mu \mathrm{M}$ in vitro (45). El artesunato inhibe el transporte de $\mathrm{Ca} 2+$ a concentraciones iguales o superiores de $2.6 \mu \mathrm{M}$, además, impide el crecimiento de $B$. bovis, lo que sugiere que podría ser utilizado como tratamiento para la babesiosis (43). La epoxomicina es un potente inhibidor de proteasoma que a concentraciones de $10 \mathrm{Nm}$ causa muerte celular en $B$. bovis, ya que promueve la ubiquitinación de proteínas en el citoplasma (46). El gossypol es una toxina natural que al inhibir la enzima lactato deshidrogenasa reduce la producción de energía de los parásitos. En cultivos in vitro de $B$. bovis fue necesario de $100 \mu \mathrm{M}$ de gossypol para inhibir su crecimiento, sin embargo, animales rumiantes presentaron efectos adversos como infertilidad en los machos, por lo que se requieren estudios sobre la eficacia de este fármaco y sus efectos tóxicos en el hospedador $(43,47)$. La atovacuona, inhibidora de la actividad deshidrogenasa afectando la biosíntesis de pirimidinas que son esenciales para la replicación del parásito, ha demostrado ser un fármaco que, aunque inhibe el crecimiento de $B$. divergens, debe estudiarse su efecto sobre otras especies de Babesia (43).

En cuanto al control del vector, este se hace mediante el uso de insecticidas en áreas de alta endemicidad y dependiendo del periodo estacional cada dos o tres semanas para tener una ventana más amplia de acción del insecticida (41). Sin embargo, se han presentado casos de resistencia debido al uso indiscriminado de los mismos y la falta de rotación en las distintas zonas, por ende, hay una reducción, pero no eliminación total de la enfermedad.

\section{Producción de vacunas}

El desarrollo de vacunas contra Babesia se ha basado en tres enfoques: el uso de parásitos vivos, atenuados o inmunógenos provenientes de ellos (48-50). Las primeras formulaciones de vacunas fueron basadas en protozoos vivos que consistían en la inoculación de sangre infectada de un portador asintomático o que hubiera resuelto la infección a animales sanos, desencadenando una respuesta inmunológica que protegía parcialmente de la infección natural (51). Sin embargo, la necesidad de mantener gran cantidad de ganado bajo condiciones libres de garrapatas, el poco uso de animales donantes infectados, la gran antelación 
con que deben ser realizados los pedidos, los altos costos de producción y, sumado a esto, la dificultad para certificar su calidad, ha provocado que varios países hayan descontinuado la producción de este tipo de vacuna (19).

Las vacunas basadas en parásitos atenuados contra $B$. bovis disminuyen la virulencia y la severidad de los signos neurológicos (52-54), sin embargo, presentan múltiples limitaciones, dentro de ellas: una vida útil corta, la reversión de la virulencia, la contaminación con otros patógenos transmitidos por la sangre, la falla en la inducción de inmunidad protectiva contra diferentes cepas y la pérdida de inmunogenicidad debido a múltiples pases sanguíneos sucesivos (9). Por otro lado, se presentan dificultades técnicas para su producción y mantenimiento, lo que resulta en un incremento de la relación costo/beneficio. Lo anterior, se debe a las siguientes razones: 1) hay que estandarizar las condiciones para mantener la atenuación de cepas y la inmunogenicidad, 2) la viabilidad es distinta según el crioprotector utilizado, el cual además puede ser tóxico, 3) tienen corta duración (4 a 7 días) y por ende se debe mantener una cadena de frío constante $\left(a 4^{\circ} \mathrm{C}\right.$ ), 4) la seguridad y protección debe ser evaluada en cada lote de producción, 5) los animales donde se produce deben estar libres de otros microorganismos para evitar la diseminación de patógenos y 6) puede ser susceptible a contaminación (51).
Con base en lo anterior, y dadas las ventajas de seguridad, eficiencia y facilidad de mantenimiento y producción, las vacunas sintéticas basadas en antígenos se están considerando para la aplicación en $B$. bovis (51). En ese orden de ideas, y teniendo claro el ciclo de vida del parásito, el punto de intervención más apropiado para el desarrollo de una vacuna es evitar su entrada a la célula; por lo cual, el estudio de moléculas parasitarias, relacionadas en la interacción con el eritrocito, es ideal en la medida en que dichas interacciones son críticas para la supervivencia de $B$. bovis dentro del hospedero.

A diferencia del enfoque clásico, se ha propuesto que más que conocer proteínas antigénicas, es ideal escoger moléculas o partes de ellas que tengan un rol importante en la unión a las células diana $(17,55)$ y, además, que sean conservadas para evitar el problema de la baja eficiencia de las vacunas actuales, asociada a la alta variabilidad genética de los parásitos, que inducen respuestas inmunes alelo-específicas (20). A pesar de que hay varios grupos que están trabajando en este campo, los reportes de caracterización de antígenos de B. bovis son pocos, resumiéndose en tan solo 7 proteínas sugeridas como candidatas a vacuna: AMA-1 (56), TRAP2 (Thrombospondin-Related Anonymous Protein) (57), MSA (Merozoite Surface Antigen1, MSA2a1, MSA2b y MSA2c) (58-60) y RAP1 $(32,61)$; AMA-1, TRAP2 y RAP 1, todas estas son conservadas en los parásitos Apicomplexa (62). Dado que 
la inmunidad adquirida depende del grado de estimulación por distintos antígenos implicados en la invasión celular, se necesita conocer y estudiar la biología del parásito en relación con las moléculas que este utiliza durante la invasión a los eritrocitos.

\section{CONCLUSIÓN}

La babesiosis bovina, particularmente aquella producida por $B$. bovis, es una enfermedad aguda asociada con la mortalidad de ganado y la disminución en la producción de distintos productos derivados de estos animales. Dado que esta enfermedad se ha convertido en una amenaza para la industria ganadera a nivel mundial, se requiere hacer una alta inversión en medidas de control con el fin de mitigar problemas para el comercio internacional del ganado.

Las estrategias disponibles para controlar la babesiosis bovina que incluyen el control del vector, el uso de medicamentos y la vacunación de ganado con cepas vivas atenuadas de $B$. bovis, hasta la fecha no han sido útiles; sin embargo, recientemente las vacunas basadas en antígenos han surgido como una estrategia alterna de prevención y tratamiento, debido a su capacidad de inducir anticuerpos que inhiben hasta en un $68 \%$ la invasión del parásito a los eritrocitos bovinos.

Por otro lado, iniciativas de estudios de ecología de vectores y de las interacciones huésped-garrapata son de importancia para mitigar el impacto en la salud animal. En esta revisión queda claro que se necesita conocer y comprender en más detalle la biología del parásito en relación con un estudio detallado de su ciclo de vida y de las moléculas implicadas en la adhesión/invasión celular. Lo anterior, permitirá caracterizar otros candidatos o moléculas involucradas en la patogénesis y evaluar su utilidad para el diseño de una vacuna contra la babesiosis causada por B. bovis.

\section{CONFLICTO DE INTERÉS}

Los autores declaran que no tienen ningún conflicto de interés.

\section{FINANCIACIÓN}

Este trabajo fue financiado por la Universidad de Boyacá.

\section{AGRADECIMIENTOS}

Queremos agradecer a la Fundación Instituto de Inmunología de Colombia (FIDIC) por permitirnos desarrollar este trabajo en la línea de investigación de Biología Molecular e Inmunología.

\section{REFERENCIAS}

1. Hunfeld K, Hildebrandt A, Gray J. Babesiosis: Recent insights into an ancient disease. Int J Parasitol. 2008;38(11):1219-37. https://doi: 10.1016/j.ijpara.2008.03.001. 
2. Bock R, Jackson $L$, De Vos A, Jorgensen W. Babesiosis of cattle. Parasitology. 2004;129(7):S247-69. https://doi: 10.1017/ s0031182004005190.

3. Gohil S, Kats LM, Sturm A, Cooke BM. Recent insights into alteration of red blood cells by Babesia bovis: moovin' forward. Trends Parasitol. 2010;26(12):591-9. https://doi: 10.1016/j. pt.2010.06.012.

4. Gray JS. Identity of the causal agents of human babesiosis in Europe. Int J Med Microbiol. 2006;296:131-6. https://doi.org/10.1016/j. ijmm.2006.01.029.

5. Nava A, Venzal J, González-Acuña D, Martins T, Guglielmone A. Ticks of the Southern Cone of America. Diagnosis, Distribution, and Hosts with Taxonomy, Ecology and Sanitary Importance. 2017.

6. ECDC. Rhipicephalus sanguineus - current known distribution: January. 2018.

7. Pérez de León AA, Strickman DA, Knowles DP, Fish D, Thacker E, de la Fuente J, et al. One Health approach to identify research needs in bovine and human babesioses: workshop report. Parasit Vectors. 2010;3(1):36. https:// doi.org/10.1186/1756-3305-3-36.
8. Suarez $\mathrm{CE}$, Noh S. Emerging perspectives in the research of bovine babesiosis and anaplasmosis. Vet Parasitol. 2011;180(1-2):109-25. https://doi: 10.1016/j.vetpar.2011.05.032.

9. Rittipornlertrak A, Nambooppha B, Simking P, Punyapornwithaya V, Tiwananthagorn S, Jittapalapong S. Low levels of genetic diversity associated with evidence of negative selection on the Babesia bovis apical membrane antigen 1 from parasite populations in Thailand. Infect Genet Evol. 2017;54: 447-54. https://doi: 10.1016/j.meegid.2017.08.009.

10. Kivaria FM. Estimated direct economic costs associated with tick-borne diseases on cattle in Tanzania. Trop Anim Health Prod. 2006;38(4):291-9. https://doi: 10.1007/ s11250-006-4181-2.

11. Bram RA, George JE, Reichard RE, Tabachnick WJ. Threat of Foreign Arthropod-Borne Pathogens to Livestock in the United States. J Med Entomol. 2002;39(3):405-16. https://doi. org/10.1603/0022-2585-39.3.405.

12. Gonzalez J, Echaide I, Pabón A, Gabriel Piñeros Jj, Blair S, Tobón-Castaño A. Babesiosis prevalence in malaria-endemic regions of Colombia. J Vector Borne Dis. 2018;55(3):222. https://doi: 10.4103/0972-9062.249480. 
13. Ríos-Osorio L, Zapata Salas R, Reyes Vélez J, Mejia J, Baena A. Enzootic Stability of Bovine Babesiosis at Puerto Berrio Region, Colombia. 2010;20(5): 485-492.

14. Vecino JAC, Echeverri JAB, Cárdenas JA, Herrera LAP. Distribución de garrapatas Rhipicephalus (Boophilus) microplus en bovinos y fincas del Altiplano cundiboyacense (Colombia). Corpoica Cienc Tecnol Agropecu. 2010;11(1):73. https://doi.org/10.21930.

15. uarez $C E$, Alzan $H F$, Silva $M G$, Rathinasamy V, Poole WA, Cooke BM. Unravelling the cellular and molecular pathogenesis of bovine babesiosis: is the sky the limit? Int J Parasitol. 2019;49(2):183-97. https://doi: 10.1016/j. ijpara.2018.11.002.

16. de Waal DT, Combrink MP. Live vaccines against bovine babesiosis. Vet Parasitol. 2006;138(1-2):88-96. https://doi.org/10.1016/j.vetpar.2006.01.042

17. Patarroyo ME, Bermúdez A, Patarroyo MA. Structural and immunological principles leading to chemically synthesized, multiantigenic, multistage, minimal subunit-based vaccine development. Chemical reviews. 2011;111(5):3459-507. https://doi: 10.1021/ cr100223m.
18. Dubremetz JF, Garcia-Réguet N, Conseil V, Fourmaux MN. Invited review Apical organeIles and host-cell invasion by Apicomplexa. Int J Parasitol. 1998;28(7):1007-13. https://doi. org/10.1016/S0020-7519(98)00076-9.

19. Kwong WK, del Campo J, Mathur V, Vermeij MJA, Keeling PJ. A widespread coral-infecting apicomplexan contains a plastid encoding chlorophyll biosynthesis. bioRxiv. 2018. https://doi.org/10.1101/391565.

20. Chauvin A, Moreau E, Bonnet S, Plantard O, Malandrin L. Babesia and its hosts: adaptation to long-lasting interactions as a way to achieve efficient transmission. Vet Res. 2009;40(2):37. https://doi: 10.1051/vetres/2009020.

21. Vannier EG, Diuk-Wasser MA, Ben Mamoun C, Krause PJ. Babesiosis. Infect Dis Clin North Am. 2015;29(2):357-70. https://doi: 10.1016/j. idc.2015.02.008.

22. OIE. World Organization for Animal Health. 2019.

23. ICA. Enfermedades de declaración obligatoria en Colombia. 2019.

24. OIE. World Organization for Animal Health. 2017. 
25. White MW, Suvorova ES. Apicomplexa Cell Cycles: Something Old, Borrowed, Lost, and New. Trends Parasitol. 2018;34(9):759-71. https://doi.org/10.1016/j.pt.2018.07.006.

26. Martinsen ES, Perkins SL, Schall JJ. A three-genome phylogeny of malaria parasites (Plasmodium and closely related genera): Evolution of life-history traits and host switches. Mol Phylogenet Evol. 2008;47(1):261-73. https:// doi: 10.1016/j.ympev.2007.11.012.

27. Allred DR, Al-Khedery B. Antigenic variation as an exploitable weakness of babesial parasites. Vet Parasitol. 2006;138(1-2):50-60. https://doi.org/10.1016/j.vetpar.2006.01.039.

28. Hines S, Mcelwain T, Buening G, Palmer G. Molecular characterization of Babesia bovis merozoite surface proteins bearing epitopes immunodominant in protected cattle. Mol Biochem Parasitol. 1989;37(1):1-9. https://doi. org/10.1016/0166-6851(89)90096-0.

29. Florin-Christensen $M$, Suarez $C E$, Hines $S A$, Palmer GH, Brown WC, McElwain TF. The Babesia bovis merozoite surface antigen 2 locus contains four tandemly arranged and expressed genes encoding immunologically distinct proteins. Infect Immun. 2002;70(7):3566-75. https://doi: 10.1128/ IAI.70.7.3566-3575.2002.
30. Bennett J, Dolin R, Blaser, M. Mandell, Douglas, and Bennett's Principles and Practice of Infectious Diseases. 2015;2.

31. Montero $\mathrm{E}$, Rodríguez $\mathrm{M}$, Oksov $\mathrm{Y}$, Lobo CA. Babesia divergens Apical Membrane Antigen 1 and Its Interaction with the Human Red Blood Cell. Infect Immun. 2009;77(11):4783-93. https://doi: 10.1128/IAl.00969-08.

32. Suarez CE, Laughery JM, Bastos RG, Johnson WC, Norimine J, Asenzo G, et al. A novel neutralization sensitive and subdominant RAP-1-related antigen (RRA) is expressed by Babesia bovis merozoites. Parasitology. 2011;138(7):809-18. https://doi: 10.1017/\$0031182011000321.

33. Salama AA, Terkawi MA, Kawai $S$, AbouLaila $M$, Nayel M, Mousa $A$, et al. Specific antibody to a conserved region of Babesia apical membrane antigen-1 inhibited the invasion of B. bovis into the erythrocyte. Exp Parasitol. 2013;135(3):623-8. http://dx.doi.org/10.1016/j.exppara.2013.09.017

34. Lobo CA, Rodriguez M, Cursino-Santos JR. Babesia and red cell invasion: Curr Opin Hematol. 2012;19(3):170-5. https://doi: 10.1097/MOH.0b013e328352245a.

35. Jalovecka M, Bonsergent C, Hajdusek O, Kopacek P, Malandrin L. Stimulation and quan- 
tification of Babesia divergens gametocytogenesis. Parasit Vectors. 2016;9(1):439. https:// doi: 10.1186/s13071-016-1731-y.

36. Mehlhorn H, Schein E. The Piroplasms: Life Cycle and Sexual Stages. En: Advances in Parasitology. Elsevier.1985;23:37-103. https://doi. org/10.1016/S0065-308X(08)60285-7

37. Howell JM, Ueti MW, Palmer GH, Scoles GA, Knowles DP. Transovarial Transmission Efficiency of Babesia bovis Tick Stages Acquired by Rhipicephalus (Boophilus) microplus during Acute Infection. J Clin Microbiol. 2007;45(2):426-31. https://doi: 10.1128/JCM.01757-06

38. Polanco Echeverry DN, Ríos Osorio LA. Aspectos biológicos y ecológicos de las garrapatas duras. Corpoica Cienc Tecnol Agropecu. 2016;17(1):81. ISSN 0122-8706

39. Mehlhorn H, Schein E. The piroplasms: "A long story in short" or "Robert Koch has seen it". Eur J Protistol. 1993;29(3):279-93. https:// doi.org/10.1016/S0932-4739(11)80371-8.

40. Jalovecka M, Sojka D, Ascencio M, Schnittger L. Babesia Life Cycle - When Phylogeny Meets Biology. Trends Parasitol. 2019;35(5):356-68. https://doi.org/10.1016/j.pt.2019.01.00
41. Yusuf J. Review on Bovine Babesiosis and its Economical Importance. Journal of Veterinary Medicine and Research. 2017;4(5):1090.

42. Echaide IE, Hines SA, McElwain TF, Suarez CE, McGuire TC, Palmer GH. In vivo binding of immunoglobulin $\mathrm{M}$ to the surfaces of Babesia bigemina-infected erythrocytes. Infect Immun. 1998;66(6):2922-7.

43. J. Mosqueda, A. Olvera-Ramírez, G. Aguilar-Tipacamú and G.J. Cantó. Current Advances in Detection and Treatment of Babesiosis. Current Medicinal Chemistry. 2012;19(10):1504-18. https://doi: 10.2174/092986712799828355.

44. Zintl A, Mulcahy G, Skerrett HE, Taylor SM, Gray JS. Babesia divergens, a bovine blood parasite of veterinary and zoonotic importance. Clin Microbiol Rev. 2003;16(4):622-36. https:// doi: 10.1128/CMR.16.4.622-636.2003.

45. AbouLaila M, Sivakumar T, Yokoyama N, Igarashi I. Inhibitory effect of terpene nerolidol on the growth of Babesia parasites. Parasitol Int. 2010;59(2):278-82. https://doi: 10.1016/j. parint.2010.02.006.

46. Meng L, Mohan R, Kwok BHB, Elofsson M, Sin N, Crews CM. Epoxomicin, a potent and selective proteasome inhibitor, exhibits in vivo antiinflammatory activity. Proc Natl Acad Sci. 
1999;96(18):10403-8. https:// doi: 10.1073/ pnas.96.18.10403.

47. Randel RD, Chase CC, Wyse SJ. Effects of gossypol and cottonseed products on reproduction of mammals. J Anim Sci. 1992;70(5):162838. https://doi.org/10.2527/1992.7051628x.

48. Elton $C M$, Rodriguez $M$, Ben Mamoun $C$, Lobo CA, Wright GJ. A library of recombinant Babesia microti cell surface and secreted proteins for diagnostics discovery and reverse vaccinology. Int J Parasitol. 2019;49(2):11525. https://doi: 10.1016/j.ijpara.2018.10.003.

49. Brown WC, Palmer GH. Designing Blood-stage Vaccines against Babesia bovis and B. bigemina. Parasitol Today. 1999;15(7):275-81. https:// doi.org/10.1016/S0169-4758(99)01471-4.

50. Laughery JM, Knowles DP, Schneider DA, Bastos RG, McElwain TF, Suarez CE. Targeted Surface Expression of an Exogenous Antigen in Stably Transfected Babesia bovis. PLoS ONE. 2014;9(5):e97890. https://doi.org/10.1371/ journal.pone.0097890.

51. Florin-Christensen $M$, Suarez $C E$, Rodriguez $A E$, Flores DA, Schnittger L. Vaccines against bovine babesiosis: where we are now and possible roads ahead. Parasitology. 2014;1-30. https://doi.org/10.1017/S0031182014000961.
52. Mangold AJ, Aguirre DH, Cafrune MM, de Echaide ST, Guglielmone AA. Evaluation of the infectivity of a vaccinal and a pathogenic Babesia bovis strain from Argentina to Boophilus microplus. Vet Parasitol. 1993;51(12):143-8. https://doi.org/10.1016/03044017(93)90205-2.

53. Mafra CL, Patarroyo JH, Silva SS. Babesia bovis: infectivity of an attenuated strain of Brazilian origin for the tick vector, Boophilus microplus. Vet Parasitol. 1994;52(1-2):139-43. https:// doi.org/10.1016/0304-4017(94)90043-4.

54. Lau AO, Kalyanaraman A, Echaide I, Palmer GH, Bock R, Pedroni MJ, et al. Attenuation of virulence in an apicomplexan hemoparasite results in reduced genome diversity at the population level. BMC Genomics. 2011;12(1):410. https:// doi: 10.1186/1471-2164-12-410.

55. Jorge S, Dellagostin OA. The development of veterinary vaccines: a review of traditional methods and modern biotechnology approaches. Biotechnol Res Innov. 2017;1(1):6-13. https://doi.org/10.1016/j.biori.2017.10.001.

56. Gaffar FR, Yatsuda AP, Franssen FFJ, de Vries E. Erythrocyte Invasion by Babesia bovis Merozoites Is Inhibited by Polyclonal Antisera Directed against Peptides Derived from a Homologue of Plasmodium falciparum Apical Membrane 
Antigen 1. Infect Immun. 2004;72(5):2947-55. https://doi: 10.1128/IAI.72.5.2947-2955.2004.

57. Terkawi MA, Ratthanophart J, Salama A, AbouLaila M, Asada M, Ueno A, et al. Molecular Characterization of a New Babesia bovis Thrombospondin-Related Anonymous Protein (BbTRAP2). Rodrigues MM, editor. PLoS ONE. 2013;8(12):e83305. https://doi.org/10.1371/ journal.pone.0083305.

58. Mosqueda J. Babesia bovis Merozoite Surface Antigen 1 and Rhoptry-Associated Protein 1 Are Expressed in Sporozoites, and Specific Antibodies Inhibit Sporozoite Attachment to Erythrocytes. Infect Immun. 2002;70(3):1599603. https://doi: 10.1128/IAI.70.3.15991603.2002.

59. Gimenez AM, Françoso KS, Ersching J, Icimoto $M Y$, Oliveira $V$, Rodriguez $A E$, et al. A recombinant multi-antigen vaccine formulation containing Babesia bovis merozoite surface antigens MSA-2a1, MSA-2b and MSA-2c elicits invasion-inhibitory antibodies and IFN- $\gamma$ producing cells. Parasit Vectors. 2016;9(1):577. https://doi: 10.1186/s13071-016-1862-1.

60. Berens SJ, Brayton KA, Molloy JB, Bock RE, Lew $A E, M c E l w a i n ~ T F$. Merozoite surface antigen 2 proteins of Babesia bovis vaccine breakthrough isolates contain a unique hypervaria- ble region composed of degenerate repeats. Infect Immun. 2005;73(11):7180-9. https:// doi: 10.1128/IAl.73.11.7180-7189.2005.

61. Yokoyama N, Suthisak B, Hirata H, Matsuo T, Inoue $N$, Sugimoto $C$, et al. Cellular localization of Babesia bovis merozoite rhoptry-associated protein 1 and its erythrocyte-binding activity. Infect Immun. 2002;70(10):5822-6. https:// doi: 10.1128/IAI.70.10.5822-5826.2002

62. Kemp LE, Yamamoto $M$, Soldati-Favre $D$. Subversion of host cellular functions by the apicomplexan parasites. FEMS Microbiol Rev. 2013;37(4):607-31. https://doi: 10.1111/15746976.12013.

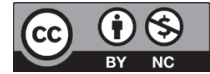

Esta obra está bajo una licencia de Creative Commons Reconocimiento-NoComercial 4.0 Internacional 